\title{
Fast prediction method of tight gas production based on segmented decline
}

\author{
Zhiqiang Fan ${ }^{1}$, Kaifeng Wen ${ }^{1}$, Tao Zhang ${ }^{1}$, Tingting Jiang ${ }^{1}$, Xiaoke Gong ${ }^{1}$, Min Jing ${ }^{2, *}$ \\ ${ }^{1}$ The Research Center of Sulige Gas-field, Changqing Oilfield Company, CNPC, Xi'an, China \\ ${ }^{2}$ College of energy, Chengdu University of science and technology, Chengdu, Sichuan, China
}

\begin{abstract}
The physical properties of tight gas reservoirs are poor. During the stable production period of gas wells, most of the recoverable reserves are produced in the declining stage. It is of great significance to accurately study the declining law of tight gas. However, tight gas production declines rapidly in the early stage and slower in the middle and late stages, and the single model fitting and prediction results have large errors. In view of the above problems, according to the production change characteristics of tight gas wells, the decline stage is divided into three stages, and a segmented production decline law analysis method is established. The research results show that the early production decline of tight gas wells is exponential decline, the relatively slow middle-term production decline belongs to depletion decline, and the late production decline belongs to linear decline. Based on the study of the law of production decline, a gas reservoir production prediction software was compiled, and the production variation characteristics of single wells and gas reservoirs under certain stable production requirements were studied.
\end{abstract}

\section{Preface}

Tight gas is one of the world's three largest unconventional natural gas resources, and it occupies an important position in the supply of natural gas energy. Due to the poor physical properties of tight gas reservoirs, the gas well enters a long decline production stage after a short stable production period. Most of the production of tight gas is produced in the decline phase. Research on the law of tight gas production decline is of great significance to the reasonable prediction of gas well production variation characteristics, EUR and the formulation of a reasonable development system.

The production decline period of tight gas wells has the characteristics of rapid decline in the early stage and slow decline in the middle and late stages. It is difficult to reasonably study the characteristics of gas well production changes using a single model. In response to the above problems, according to the tight gas production change characteristics, the tight gas decline stage is divided into three stages, and the segmented decline law analysis method is used to carry out the production decline research. Through example gas wells, the production variation characteristics of tight gas wells in different production stages are analyzed. Based on the production variation characteristics of tight gas, production prediction software is compiled to predict the production of tight gas reservoirs.

\section{Variation characteristics of tight gas single well production}

The production changes of gas wells during the production process show obvious two-stage characteristics. The early pressure drop is fast. This stage is in the early stage of instability, mainly based on the linear flow of fracturing fractures, and the pressure drop rate is large; the second stage is the natural continuous production stage, the pressure drop rate remains stable, the production decline is slow, and the gas well production begins to reach the level of reserves production.

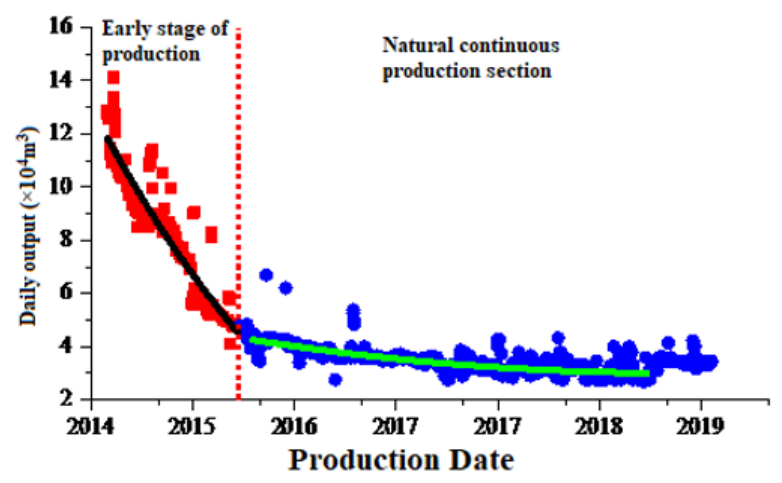

Figure 1. Division of production stages of tight gas wells

From the fitting results of actual production data of Well A (Figure 1), the three traditional decline models cannot

\footnotetext{
* Corresponding author: 2020050162@stu.cdut.edu.cn
} 
fit well the production history of Well A, and the predicted annual gas production in the later stage of production is consistent with the actual value. Inconsistent, the annual gas production predicted by different declining methods is higher or lower.

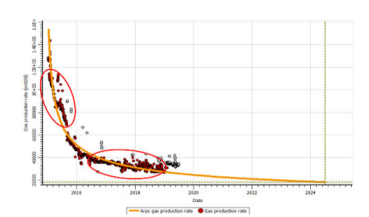

(1) Arps decreasing fitting

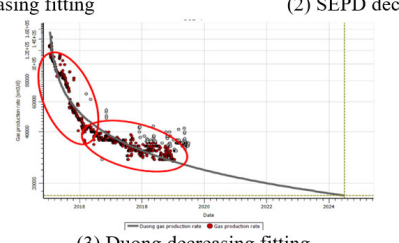

(3) Duong decreasing fitting

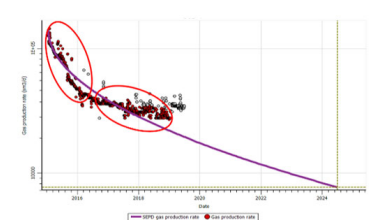

(2) SEPD decreasing fitting
Figure 2. Fitting effects of different decreasing methods

It is imperative to carry out research on the law of stepwise decline in tight gas wells to reasonably predict the production of gas wells.

\section{Stepwise decline model for tight gas wells}

According to the seepage characteristics of tight gas wells and the production characteristics of gas wells, the life cycle of gas wells is divided into three stages: early stage, middle stage and late stage. In the first two stages, gas wells produced more than $80 \%$ of cumulative gas production. During the production process of gas wells, the first two stages of gas well management were emphasized, as shown in Figure 3. The staged evaluation of the decline rate change characteristics is a major feature of tight gas well production.

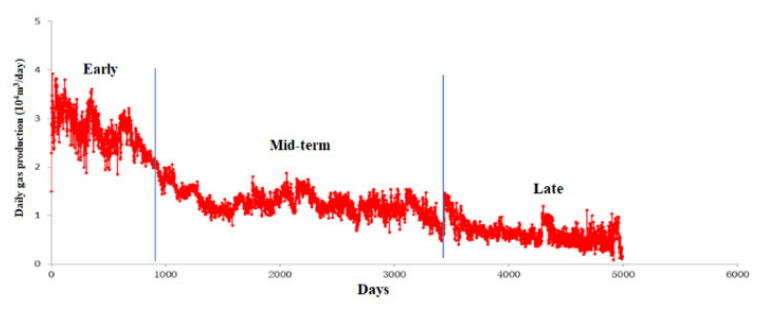

Figure 3. Schematic diagram of gas well life cycle division

In the early stage of production of tight gas wells, the production declines quickly, and the production time is 13 years. The initial production of gas wells is relatively high, the production declines quickly, and the decline rate is large. After multiple decline models are fitted, the early production meets the exponential decline model, but if the entire stage All using the exponential decline model, the later stage output of the model is much lower than the time output, which does not meet the characteristics of tight gas production, as shown in Figure 4.

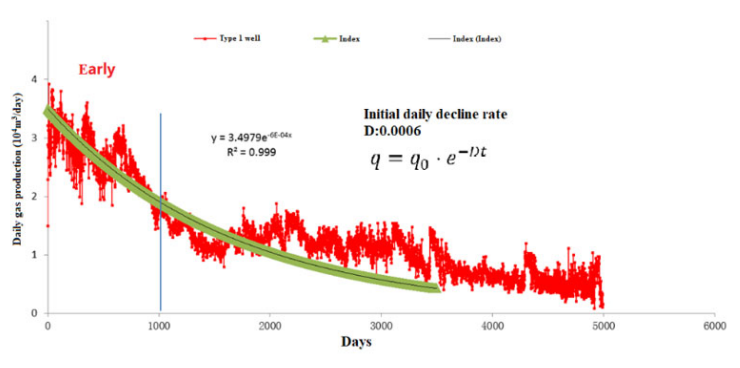

Figure 4. The law of production decline in the early stage of tight gas wells

After the gas well enters the mid-term, the decline rate slows down, the production lasts 4-10 years, and the relatively stable production phase begins for a long time, and the production pressure declines slowly. At this time, the decline rate change law conforms to the exhaustion decline, as shown in Figure 5.

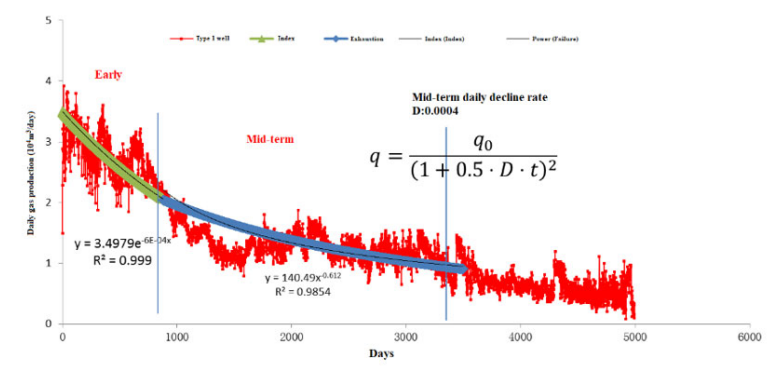

Figure 5. The law of mid-term production decline of tight gas wells

After the gas well enters the late stage, tight gas wells need indirect separation system or drainage gas recovery to obtain better productivity. The rate of gas well opening is less than $50 \%$. If the early and mid-term decline model is used, the fitted production rate is higher than the actual gas production rate. . After analysis, it is recommended to use the change of annual gas production over time to evaluate the later decline rate, as shown in Figure 6. The prediction results show that the late decline law of tight gas wells conforms to the characteristics of the linear decline model.

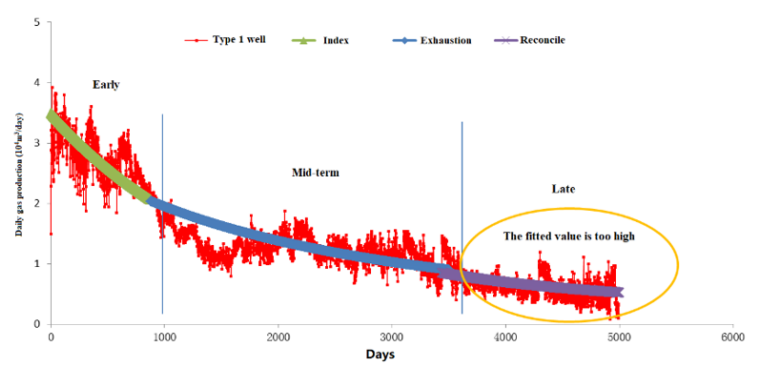

Figure 6. The law of late production decline of tight gas wells

\section{Compilation of tight gas reservoir production prediction software}

The production stability of tight gas wells is poor, so the mode of supplementing new wells is often used in the development of tight gas reservoirs to ensure that the gas reservoirs can produce stable gas production at a certain 
scale. Therefore, on the basis of the study of tight gas well production decline law, the production forecasting software was compiled using VBA program to carry out the production forecasting work of a tight gas reservoir in China. The tight gas production forecast process is as follows:

1) Determine the number of development wells based on parameters such as geological reserves, gas-bearing area, reasonable well spacing, and production indicators;

2) Comply with the top-valley-bottom peak "bright spot" model of high-quality reservoir seismic response mode, and determine high-quality reservoir targets based on the strong peak reflection mode, deploy horizontal wells, and the horizontal section is perpendicular to the maximum horizontal principal stress direction, which is beneficial to the reservoir Transformation;

3) Determine the number of replacement wells for annual gas production based on the decline in gas wells and the production value of a single well;

4) Finally, according to the number of deployed wells, the gas field gas production change characteristics, cumulative gas production and production degree and other characteristic parameters are determined, and the block development potential prediction and development index prediction work are carried out.

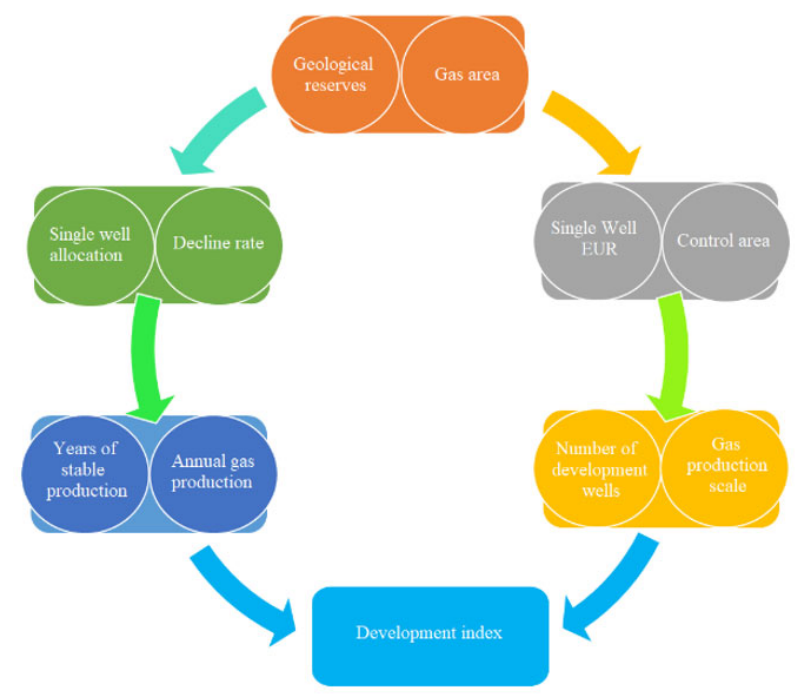

Figure 7. Key indicators deployment ideas

Enter the target scale, single well production allocation, single well stable production period, abandoned production, production start time, production end time, predicted age, gas-bearing area, single well control area, well construction rate, geological reserves and drilling in the software Number and other parameters. The gas production changes of a single well are calculated separately as shown in Figure 8.

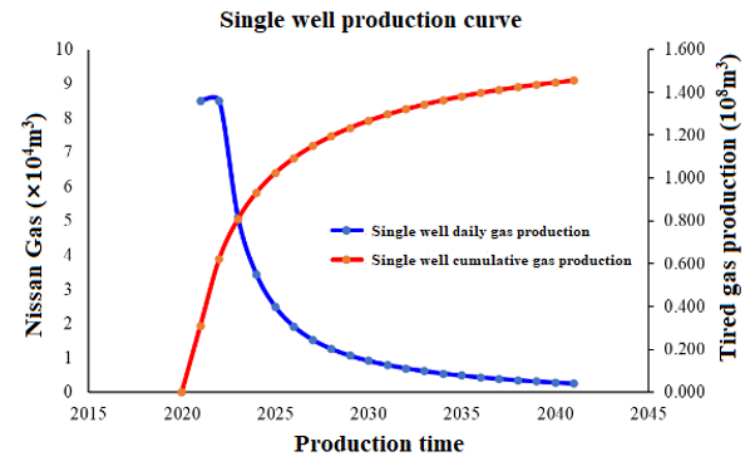

Figure 8. Predicted production curve

The tight gas reservoir has been producing stable production for 10 years with an annual output of 2.5 billion cubic meters. The gas production volume of the gas reservoir over the years and the production contribution of different types of gas wells are shown in Figure 9.

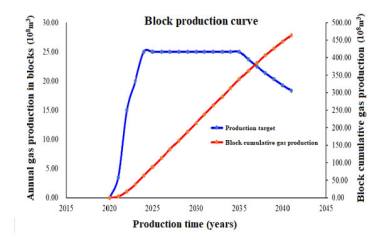

(1) Prediction of gas production in gas reservoir

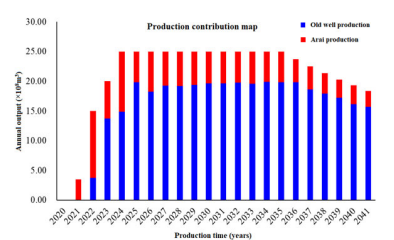

(2) Production contribution of different types of
Figure 9. Block production and production contribution characteristics of different gas wells

Due to the rapid decline in production of tight gas wells, the decline rate of gas reservoirs is higher after the 10year stable production period. In the process of gas reservoir development, with the increase in the number of wells, the contribution of old wells to gas reservoir production continues to increase.

\section{Conclusion}

(1) The production change of tight gas wells presents multi-stage characteristics, with rapid production decline in the early stage and slower production decline in the middle and late stages. The use of a single decline model to analyze the law of production decline has large errors.

(2) According to the production characteristics of tight gas wells, the production decline of tight gas wells is divided into three stages. The ARPS decline method can be used for fitting to better analyze the production decline characteristics of tight gas wells. The early decline of gas wells conforms to the exponential law, and the mediumterm production decline conforms to failure. Decrease model, the law of late decline conforms to the linear decline model.

(3) According to the tight gas decline characteristics, the tight gas reservoir production prediction software was compiled using VBA, and the production change characteristics of a single well and the entire gas reservoir were predicted. 


\section{References}

1. Chen Yuanqian, Fu Libing, Xu Jiaqian. Application and comparison of two types of production decline models in the prediction of shale gas wells and tight gas wells[J/OL]. Petroleum Geology and Recovery Efficiency: 1-6 [2021-11-23] .http://kns.cnki.net/ $\mathrm{kcms} /$ detail/ 37.1359. TE. 20201210. 1720.002.html.

2. Chen Yuanqian, Fu Libing, Xu Jiaqian. Application and comparison of two types of production decline models in the prediction of shale gas wells and tight gas wells[J]. Petroleum Geology and Recovery Efficiency, 2021, 28(03): 84-89.

3. Li Yanzun, Bai Yuhu, Xu Bingxiang, Chen Ling, Dong Zhiqiang. The application of NARX neural network in the production performance prediction of tight gas wells [A]. Xi'an Shiyou University, Chengdu University of Technology, Shaanxi Petroleum Institute. 2020 Oil and Gas Field Exploration and Proceedings of Development International Conference[C]. Xi'an Shiyou University, Chengdu University of Technology, Shaanxi Petroleum Society: Xi'an Shiyou University, 2020: 8.

4. Wang Xilin. Research on productivity prediction of fractured horizontal wells in anisotropic tight gas reservoirs[D]. China University of Geosciences (Beijing), 2020.

5. Liang Yiwei, Wang Dong, Li Tingjun, Du Chao. Variation characteristics of Arps production decline index for tight gas wells[J]. Journal of Xi'an Shiyou University (Natural Science Edition), 2018, 33(02): 77-81.

6. Wei Yunsheng, Jia Ailin, He Dongbo, Wang Junlei, Han Pinlong, Jin Yiqiu. Development characteristics and development technology similarities and differences between shale gas and tight gas in China[J]. Natural Gas Industry, 2017, 37(11): 43-52.

7. Yang Zhen, Kong Lingfeng, Du Min, Zhao Chenhui. Economic evaluation of domestic tight sandstone gas development projects and fiscal and taxation support policy research[J]. Natural Gas Industry, 2016, 36(07): 98-109.

8. Wang Cai. Research on productivity calculation method for fractured gas wells in tight gas reservoirs[D]. China University of Geosciences (Beijing), 2016.

9. Hu Junkun, Gong Wei, Ren Ke. Analysis of key factors and countermeasures for China's tight gas development[J]. Natural Gas Technology and Economy, 2015, 9(06): 24-29+82.

10. Li Chen, Xia Chaohui, Wang Ping, Zhao Yixuan, Zhang Hongyan. Application of average decline index in tight gas reserve evaluation[J]. Special Oil and Gas Reservoirs, 2015, 22(02): 105-107+156.

11. Wang Xin, Qi Mei, Hu Yongle. Application of average model in production prediction of tight gas wells[J]. Science Technology and Engineering, 2015, 15(07): 173-176+180.
12. Gu Ning, Zhu Xueqian, Xing Liwen. Analysis of calculation methods for unconventional gas reserves[J]. Natural Gas and Petroleum, 2014, 32(06): 45-49+11.

13. Li Jiangfei, Fu Zhizhong, Zhang Huanzhi, Li Bo. Tight gas development and production prediction based on technological progress and engineering economic models [A]. Xi'an Shiyou University, Shaanxi Petroleum Institute, Xi'an Petroleum Service Industry Alliance. 2013 Oil and Gas Reservoir Monitoring And Management International Conference and Exhibition Proceedings[C]. Xi'an Shiyou University, Shaanxi Petroleum Institute, Xi'an Petroleum Service Industry Alliance: Xi'an Huaxian Network Information Service Co., Ltd., 2013: 7 .

14. Qiu Zhongjian, Zhao Wenzhi, Deng Songtao. Development prospects and strategic significance of tight sandstone gas and shale gas in China[J]. China Engineering Science, 2012, 14(06): 4-8+113.

15. Yang Tao, Zhang Guosheng, Liang Kun, Zheng Min, Guo Bincheng. Global tight gas exploration and development progress and China's development trend forecast [J]. Chinese Engineering Science, 2012, 14(06): 64-68+76. 